\title{
Expression of Th, Comt and Maoa genes and brain catecholamine levels in rats with genetic catatonia
}

\author{
M. Ryazanova ${ }^{1,3 *}$, T. Latysheva ${ }^{2}$, V. Plekanchuk ${ }^{1,3}$, O. Prokudina ${ }^{1}$, M. Gilinsky ${ }^{2}$, \\ T. Alekhina ${ }^{1}$ \\ ${ }^{1}$ Institute of Cytology and Genetics SB RAS, Novosibirsk, Russia \\ ${ }^{2}$ Research Institute of Physiology and Fundamental Medicine, Novosibirsk, Russia \\ ${ }^{3}$ Novosibirsk State University, Novosibirsk, Russia \\ *e-mail:ocean-2006@yandex.ru
}

Key words: genetic catatonia, genes expression, catecholamines

Motivation and Aim: The work was performed on the GC rats strain with genetic catatonia, which can be considered as a model of the catatonic syndrome observed in schizophrenia, affective disorders and other diseases [1]. It is known that brain dopaminergic system is involved in development of catatonia and schizophrenia and its involvement is complex. Many drugs for treatment of psychosis have an effect on dopamine system function. The noradrenergic system can modulate dopamine system, as well as directly affect on motor activity and emotional sphere. The aim of this work was to study mRNA expression of genes catecholamines synthesis - tyrosine hydroxylase (Th) and degradation - catecholO-methyltransferase (Comt) and monoamine oxidase A (Maoa), and also to estimate the catecholamines and 3,4- dihydroxyphenylacetic acid (DOPAC) content in brain structures in inbred GC rats.

Methods and Algorithms: Using RT-qPCR Th, Comt, Maoa mRNA level in brain structures (hypothalamus, striatum, amigdala, midbrain, medulla oblongata) of GC an WAG rats was estimated. High performance liquid chromatography with electrochemical detection was used for investigation the level of catecholamines (dopamine and noradrenaline) and DOPAC in same brain structures.

Results: The study revealed a low level of Maoa mRNA in striatum of rats with genetic catatonia. The mRNA level of Comt, Th in brain structures GC rats did not differ from the control. The high level of norepinephrine in hypothalamus, midbrain and striatum of GC rats was shown.

Conclusion: The high level of norepinephrine in striatum of GC rats may be due to the low mRNA expression of Maoa in this structure, however, the increase norepinephrine level in the hypothalamus and in midbrain are not associated with the altered expression of the mRNA gene. The revealed changes in the level of norepinephrine in the brain can contribute to the increased excitability and nervosity of GC rats, which they shown before [2] along with the akinetic reactions of freezing.

Acknowledgements: Supported by the RFBR (grant No. 16-04-01325).

\section{References}

1. Kolpakov V.G. et al. (2004) Catatonia or depression? The GC rat strain is a genetic animal model of psychopathology. Genetics. 40(6):827-834.

2. Ryazanova M.A. (2012) Increase in the share of "nervous" animals during the selection for catatonia: participation in catatonic reactions of central adrenoreceptors. Genetics. 48(11):1141-1147. 